\title{
QUASI-PERIODIC SOLUTIONS OF NONLINEAR ORDINARY DIFFERENTIAL EQUATIONS WITH SMALL DAMPING
}

\author{
BY MARCELLE FRIEDMAN
}

Communicated by F. John, December 15, 1966

A function $f(t)$ is called quasi-periodic if it can be represented in the form

$$
f(t)=F\left(\omega_{1} t, \omega_{2} t, \cdots, \omega_{m} t\right)
$$

where $F\left(\theta_{1}, \cdots, \theta_{m}\right)$ is a continuous function of period $2 \pi$ in $\theta_{\nu}$, $\nu=1, \cdots, m$. The numbers $\omega_{1}, \cdots, \omega_{m}$ are called the basic frequencies of $f(t)$. We shall denote by $A\left(\omega_{1}, \cdots, \omega_{m}\right)$ the class of all functions $f$ for which $F$ is real analytic. For simplicity of notation we set $\theta=\left(\theta_{1}, \cdots, \theta_{m}\right)$ and $\omega=\left(\omega_{1}, \cdots, \omega_{m}\right)$ (then $A\left(\omega_{1}, \cdots, \omega_{m}\right)=A(\omega)$ and $\left.F\left(\theta_{1}, \cdots, \theta_{m}\right)=F(\theta)\right)$.

The purpose of this note is to study the family of complex systems of differential equations:

$$
\begin{aligned}
& \dot{z}=\lambda z+\epsilon f(t, z, \bar{z}), \\
& \dot{\theta}=\omega
\end{aligned}
$$

parametrized by $\lambda, f$ analytic in $z, \bar{z}$, and $f \in A(\omega)$-i.e. $f(t, z, \bar{z})$ $=g(\theta, z, \bar{z})$ where $g$ is $2 \pi$-periodic in $\theta$-to determine the complex numbers, $\lambda$, for which there exists a solution $z=\phi(t, \epsilon) \in A(\omega){ }^{1}$

For $\operatorname{Re} \lambda=0$ there may be no solutions even in the linear case

$$
\begin{aligned}
& \dot{z}=\lambda z+\epsilon g(\theta), \\
& \dot{\theta}=\omega
\end{aligned}
$$

because of resonance. It is well known that if $\operatorname{Re} \lambda \neq 0$ and $\epsilon>0$ is small compared with $|\operatorname{Re} \lambda|$ then (1) always has a solution $z=\phi(t, \epsilon)$ $\in A(\omega)$. This was shown by Malkin [7] and Bohr and Neugebauer [4] in the linear case and by Stoker [10] and, in the general case, by Bogoliubov [1].

Our main interest is $|\operatorname{Re} \lambda|$ small compared to $\epsilon$. We shall describe a domain, $\Omega$, in the $\lambda$-plane such that for each $\lambda \in \Omega$ the corresponding system (1) has a solution $z=\phi(t, \epsilon) \in A(\omega)$. We call $\Omega$ a nonresonance domain. We will show that $\Omega$ contains in particular $|\operatorname{Re} \lambda|>1$ (this

1 This system is derived from the second order equation $\ddot{x}+c \dot{x}+a x=f(t, x, \dot{x})$ ( $f$ quasi-periodic in $t$ ) by the transformation $z=\dot{x}+\alpha x$ for some constant $\alpha$. 
corresponds to the above-mentioned result of Bogoliubov) and in the remaining strip consists of a collection of closed sets each connecting the two half planes which we will call $\Omega_{+}$and $\Omega_{-}$. (See Figure 1.) Moreover, the complement of these closed sets has small measure, independent of $\epsilon$.

We set $(\omega, k)=\sum_{\nu=1}^{m} \omega_{\nu} k_{\nu}$ where the $k_{\nu}, \nu=1, \cdots, m$ are integers, and $|k|=\sum_{\nu=1}^{m}\left|k_{\nu}\right|$. If we assume that $g$ is analytic for $|z|,|\bar{z}|<r$, $|\operatorname{Im} \theta|=\sum\left|\operatorname{Im} \theta_{\nu}\right|<1$ and that $|g|<1$, then

THEOREM. If $|(\omega, k)| \geqq c_{0}^{-1}|k|^{-\tau}, c_{0}>1, \tau>m$, then there exists $\epsilon_{0}=\epsilon_{0}(m)$ such that for $\epsilon \leqq \epsilon_{0}$ there exists a closed, connected set, $\Omega=\Omega(\epsilon)$ in the $\lambda$-plane such that for the corresponding system

$$
\begin{aligned}
& \dot{z}=\lambda z+\epsilon g(\theta, z, \bar{z}), \\
& \dot{\theta}=\omega
\end{aligned}
$$

has a solution

$$
z=\phi(t, \epsilon) \in A(\omega) .
$$

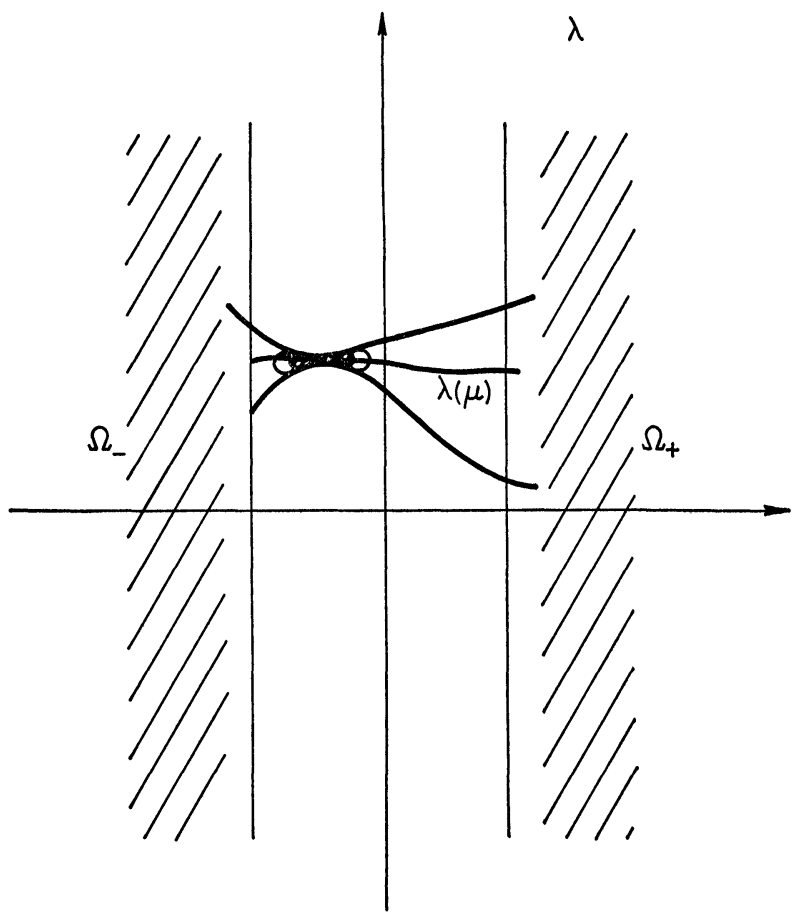

FIGURE 1 
The set $\Omega$ contains the half planes $\Omega_{+}$and $\Omega_{-}$. The latter two sets are connected by infinitely many cusp-like domains bounded by curves with one point of contact. The quasi-periodic solutions are stable or unstable according as $\lambda$ lies to the left or right of the contact point.

It should be noted here that although $\Omega$ depends on $\epsilon$ and $g$ the measure of the complement is small independent of the perturbation. This implies that for most choices of $\lambda$ the system $(1)^{\prime}$ has a solution $\in A(\omega)$.

The complement of $\Omega$ is not empty even in the linear case. This can be seen as follows:

We find a solution $z$ of the linear problem by means of Fourier series. Substituting in $\dot{z}=\lambda z+\epsilon g(\theta)$ we obtain the following equations for the Fourier coefficients $z_{k}$ of $\boldsymbol{z}$ :

$$
\{i(\omega, k)-\lambda\} z_{k}=\epsilon g_{k} .
$$

For $\operatorname{Re} \lambda=0,|i(\omega, k)-\lambda|$ can be arbitrarily small since $\omega_{1}, \cdots, \omega_{m}$ are rationally independent. To prevent this we must restrict the choice of $\lambda$. If we require that $\lambda$ satisfy the inequality $|i(\omega, k)-\lambda| \geqq \gamma^{-1}$ for some constant $\gamma>1$, we find that all pure imaginary $\lambda$ are excluded. However, if we weaken the condition to

$$
|i(\omega, k)-\lambda| \geqq\left(\gamma|k|^{\tau}\right)^{-1}
$$

where $\gamma>1, \tau>m$, we find that the measure of the excluded set on any line parallel to the imaginary axis is proportional to $\gamma^{-1}$ and decreases as $|\operatorname{Re} \lambda|$ increases. Hence there are pure imaginary $\lambda$ for which $\dot{z}=\lambda z+\epsilon g(\theta)$ has a formal solution $z$ (convergence is assured if $g(\theta)$ is sufficiently differentiable).

The proof of our theorem is divided into two steps. The first and main step will consist of finding a family of curves, $\Gamma$, in the $\lambda$-plane such that for the corresponding differential equation we can

(i) construct quasi-periodic solutions belonging to $A(\omega)$,

(ii) transform the linearized equation (linearized on these solutions) in to constant coefficients.

If $|\operatorname{Re} \lambda|>1$ we can easily use the contraction principle on the iteration scheme

$$
\begin{aligned}
z_{0} & =0, \\
\dot{z}_{n+1}-\lambda z_{n+1} & =\epsilon g\left(\theta, z_{n}, \bar{z}_{n}\right)
\end{aligned}
$$

and show convergence for $\epsilon /|\operatorname{Re} \lambda|$ sufficiently small. (This is essen- 
tially the technique of Bogoliubov [2], Stoker [10], and Malkin [7].) Our main interest, however, is for $|\operatorname{Re} \lambda|$ small. Here we need the "rapid convergence" technique of Kolmogorov [5], [6], Arnol'd [1], and Moser [8]. More precisely, we proceed as follows.

We construct a quasi-periodic transformation $z=\zeta+v(\theta, \zeta, \bar{\zeta}, \lambda)$ taking $(1)^{\prime}$ into

$$
\begin{aligned}
& \dot{\zeta}=\mu \zeta+\phi(\theta, \zeta, \bar{\zeta}, \mu)=\mu \zeta+\theta\left(|\zeta|^{2}\right), \\
& \dot{\theta}=\omega
\end{aligned}
$$

where $\mu$ satisfies

$$
\begin{aligned}
\left|(\omega, k)-j_{0} \operatorname{Im} \mu\right| \geqq(\gamma|k| \tau)^{-1}, & \\
\gamma & >1, \quad \tau>m, \quad|k| \neq 0, \quad j_{0}=0,1,2 .
\end{aligned}
$$

This provides a quasi-periodic solution $z=v(\omega t, 0,0, \lambda) \in A(\omega)$ on a nondenumerable set of curves connecting $\Omega_{+}$and $\Omega_{-}$.

In the second step of the proof, in order to enlarge the domain we must give up the requirement that the linearized equation be transformable into constant coefficients. For every $\mu$ with $\operatorname{Re} \mu \neq 0$ using a contraction argument we can ensure the existence of a solution $z \in A(\omega)$ if $\lambda$ is sufficiently close to the above determined curves, $\lambda=\lambda(\mu)$. It suffices to take $|\lambda-\lambda(\mu)|<c|\operatorname{Re} \mu|^{2}$. This determines for each curve in $\Gamma$ a parabolic neighborhood (see Figure 1) with point of contact at $\operatorname{Re} \mu=0$.

It should be noted here that the point of contact need not be on $\operatorname{Re} \lambda=0$. However, for reversible systems $(g(\theta, z, \bar{z})$ $=[-\bar{g}(-\theta,-\bar{z},-z)])$ it was shown by Moser [9] that all contact points lie on $\operatorname{Re} \lambda=0$.

\section{BIBLIOGRAPHY}

1. V. I. Arnol'd, Small divisors and stability problems in classical and celestial mechanics, Uspehi Mat. Nauk SSSR 18, ser. 6 (114), (1963), 81-192. (Russian)

2. N. N. Bogoliubov, On some statistical methods of mathematical physics, Izv. Acad. Nauk SSSR. 1945. (Russian)

3. - On quasi-periodic solutions in nonlinear problems of mechanics, Lectures held at the First Mathematical Summer School, Kanev, 1963, Akad. Nauk, Ukrain. SSSR, 1964.

4. H. Bohr and O. Neugebauer, Über lineare Differential-gleichungen mit konstanten Koeffizienten und fast-periodischen rechter Seite, Nachr. Akad. Wiss. Göttingen, Math. phys. K1 1926, pp. 8-22.

5. A. N. Kolmogorov, Dokl. Akad. Nauk. SSSR 98 (1954), 527-530.

6. - General theory of dynamical systems and classical mechanics, Vol. 1, pp. 315-333, Proc. Internat. Congress of Math., Amsterdam, 1954, Amsterdam: Nordhoff, Amsterdam, 1957. 
7. I. G. Malkin, Some problems in the theory of nonlinear oscillations, State Publishing House, Moscow, 1956.

8. J. Moser, A new technique for the construction of solutions of nonlinear differential equations, Proc. Nat. Acad. Sci., U.S.A. 47 (1961), 1824-1831.

9. - Combination tones for Duffing's equation, Comm. Pure Appl. Math. 18 (1965), 167-181.

10. J. J. Stoker, Nonlinear vibrations, Interscience, New York, 1950, pp. 235-239.

Seton Hall University 